\title{
Ferromagnetic resonance and magnetooptic study of submicron epitaxial $\mathrm{Fe}(001)$ stripes
}

\author{
E. Paz, ${ }^{1}$ F. Cebollada, ${ }^{2, a)}$ F. J. Palomares, ${ }^{1}$ J. M. González, ${ }^{3}$ J. S. Martins,${ }^{4}$ N. M. Santos, ${ }^{4}$ \\ and N. A. Sobolev ${ }^{4}$ \\ ${ }^{1}$ Instituto de Ciencia de Materiales de Madrid-CSIC, c/Sor Juana Inés de la Cruz, 3, 28049 Madrid, Spain \\ ${ }^{2}$ POEMMA-CEMDATIC, ETSI Telecomunicación, Universidad Politécnica de Madrid, 28040 Madrid, Spain \\ ${ }^{3}$ Unidad Asociada ICMM-IMA, c/Sor Juana Inés de la Cruz, 3, 28049 Madrid, Spain \\ ${ }^{4}$ Departamento de Física and I3N, Universidade de Aveiro, 3810-193 Aveiro, Portugal
}

(Received 2 April 2012; accepted 21 May 2012; published online 27 June 2012)

\begin{abstract}
We present a combined magnetooptic and ferromagnetic resonance study of a series of arrays of single-crystalline $\mathrm{Fe}$ stripes fabricated by electron beam lithography on epitaxial $\mathrm{Au}(001) / \mathrm{Fe}(001) /$ $\mathrm{MgO}(001)$ films grown by pulsed laser deposition. The analysis of the films revealed a clear fourfold magnetocrystalline anisotropy, with no significant presence of other anisotropy sources. The use of a large series of arrays, with stripe widths between 140 and $1000 \mathrm{~nm}$ and separation between them of either $200 \mathrm{~nm}$ or $500 \mathrm{~nm}$, allowed studying their magnetization processes and resonance modes as well as the effects of the dipolar interactions on both. The magnetization processes of the stripes were interpreted in terms of a macrospin approximation, with a good agreement between experiments and calculations and negligible influence of the dipolar interactions. The ferromagnetic resonance spectra evidenced two types of resonances linked to bulk oscillation modes, essentially insensitive to the dipolar interactions, and a third one associated with edge-localized oscillations, whose resonance field is strongly dependent on the dipolar interactions. The ability to produce a high quality, controlled series of stripes provided a good opportunity to achieve an agreement between the experiments and calculations, carried out by taking into account just the Fe intrinsic properties and the morphology of the arrays, thus evidencing the relatively small role of other extrinsic factors. @ 2012 American Institute of Physics. [http://dx.doi.org/10.1063/1.4730136]
\end{abstract}

\section{INTRODUCTION}

Magnetic nanostructures are quite relevant for technological applications related with magnetoelectronics, radiofrequency devices, and magnetic storage. ${ }^{1-3}$ In order to implement actual devices, an excellent control of their static magnetization processes and of the high frequency magnetization dynamics is necessary, which, in turn, requires a good control of the magnetic anisotropy and of the internal effective fields. Nanoelements based on epitaxial Fe thin films, usually grown on insulators and semiconductors, such as $\mathrm{MgO}, \mathrm{GaAs}$, InAs, $\mathrm{ZnSe}$, or $\mathrm{Si}$, are among the most widely studied due to their high magnetization and to the possibility of choosing the relative orientation of the crystallographic axes with respect to the axes defining the shape of the nanoelements, which provides an easy way to control two of the main contributions to the anisotropy. ${ }^{4-7}$ However, the coupling of the magnetization to structural features or defects usually gives rise to other non-negligible extrinsic sources of anisotropy. In most cases, such a coupling occurs due to specificities of the deposition or lithography processes, to the interface between the substrate and the film, and even to the capping layer commonly used to avoid the oxidation of the latter. The presence of steps or structures with two-fold symmetry on the reconstructed surfaces of the substrates usually gives rise to a uniaxial component of the anisotropy of

\footnotetext{
a) Author to whom correspondence should be addressed. Electronic mail: fcebollada@etsit.upm.es.
}

the Fe film, which in some cases is significant even for films tens of nanometers thick. ${ }^{89}$ The roughness or intermixing of chemical species at the film-substrate interface and the magnetoeleastic interactions due to the lattice mismatch may also become important sources of anisotropy. ${ }^{10,11}$ Last but not least, the interface between the capping layer and the film has also proven to provide an extra contribution, which may modify the magnetization processes. ${ }^{12}$

Ferromagnetic resonance (FMR) is based on the precession of the magnetization around an effective field. The resonance absorption is induced by an rf field typically in the range of gigahertz, and the measurements are usually carried out by sweeping the polarizing magnetic field applied to a sample located inside a resonance cavity. FMR measurements in saturation conditions (uniform mode of magnetization precession) are highly sensitive to the effective field inside nanoscaled samples, which allows studying different contributions to the magnetic anisotropy. Under these conditions, the resonance linewidth yields information about the relaxation processes arising from the intrinsic damping and extrinsic factors due to structural features such as surface roughness and mosaicity. ${ }^{13}$ Finally, the FMR techniques may also provide information about different types of spin excitations that can occur in nanoelements, basically quantized spin waves due to confinement effects ${ }^{14}$ or localized modes generated by nonuniform internal fields. ${ }^{15-18}$

In this work, we report on the static and dynamic magnetization of a series of epitaxial $\mathrm{Fe}(001) / \mathrm{MgO}(001)$ films and arrays of stripes with submicron width and separation. By 
means of a combination of magnetooptic and FMR techniques, we have been able to separate different contributions to the magnetic anisotropy and resonances to which they are linked, as well as to reveal the influence of dipolar interactions between the stripes. Good agreement between calculations and experiments has evidenced the negligible influence of extrinsic anisotropy sources related to the interfaces or to the lithography processes on the magnetic behaviour, which is a consequence of the high crystalline quality of the samples.

\section{SAMPLES AND EXPERIMENTAL CONDITIONS}

A series of $\mathrm{Fe}(001)$ films were deposited on $\mathrm{MgO}(001)$ substrates under UHV conditions by pulsed laser deposition (PLD). Prior to deposition, the substrates were thermally treated at $200{ }^{\circ} \mathrm{C}$ for $25 \mathrm{~min}$ for water desorption. After deposition, the films were annealed at $400{ }^{\circ} \mathrm{C}$ for $25 \mathrm{~min}$ in order to improve their crystalline quality and to decrease the surface roughness. A $5 \mathrm{~nm}$ thick $\mathrm{Au}(001)$ oxidation-protection capping layer was subsequently deposited on top of the films at room temperature (RT) by molecular beam epitaxy.

The crystallinity, structure, and quality of the interfaces between the different layers of the samples were studied by means of the X-ray diffraction and reflectivity (XRD and XRR, respectively) using both conventional (Brucker D8 Advance diffractometer with $\mathrm{Cu}-\mathrm{K} \alpha$ radiation, $\lambda=1.5418 \AA$ ) and synchrotron (BM25 beamline of the European Synchrotron Radiation Facility, ESRF, $14 \mathrm{keV}, \lambda=0.8857 \AA$ ) radiation sources. The $\mathrm{X}$-ray characterization, already published, ${ }^{4}$ evidenced a strong texture, with the (001) direction of both the $\mathrm{Fe}$ and $\mathrm{Au}$ layers perpendicular to the plane of the films, as well as the existence of a single Fe crystalline domain with the Fe lattice rotated $45^{\circ}$ with respect to that of the $\mathrm{MgO}$ substrate, the epitaxial relation thus being $\mathrm{Fe}(001)[100] / / \mathrm{MgO}(001)[110]$. The thicknesses of the $\mathrm{Fe}$ and $\mathrm{Au}$ layers, calculated from the oscillation periods of the XRR scans, are $24 \mathrm{~nm}$ and $3 \mathrm{~nm}$, respectively. The surface morphology of the samples was analysed by atomic force microscopy (AFM), which evidenced very smooth surfaces with a mean roughness under $5 \AA$.

Arrays of $100 \mu \mathrm{m}$ long stripes, having widths $(w)$ in the range between $140 \mathrm{~nm}$ and $1000 \mathrm{~nm}$ and separations (d) between the wires of either $200 \mathrm{~nm}$ or $500 \mathrm{~nm}$, were fabricated on the $\mathrm{Fe} / \mathrm{Au}$ films by means of electron beam lithography (EBL) at the Nano-Bio Center of the Technical University of Kaiserslautern. All the arrays were fabricated with the [100] and [010] axes of the Fe layer oriented parallel and perpendicular to the long axis of the stripes, respectively.

Magnetooptic Kerr effect (MOKE) and FMR techniques were employed to characterize the magnetic behaviour of the continuous films and stripe arrays. A MOKE device simultaneously operated in both transverse and longitudinal configurations allowed to study the angular dependence of the hysteretic processes, yielding both the parallel- and transverse-to-the-field components of the magnetization, in applied fields of up to $5 \mathrm{kOe}$ at RT. FMR measurements were done, also at RT, in a Bruker ESP $300 \mathrm{E}$ X-band $(\sim 9.85 \mathrm{GHz})$ electron paramagnetic resonance spectrometer using a $\mathrm{TE}_{102}$ resonant cavity and magnetic fields up to 1.5 T. The microwave magnetic field was always oriented normally to the static magnetic field. The samples were driven through the resonance by sweeping and modulating the latter field, so that the FMR signal, measured via lock-in techniques, was proportional to the first derivative of the imaginary part of the susceptibility. A goniometer with $0.25^{\circ}$ resolution allowed rotating the sample around an axis perpendicular to its plane in order to study the in-plane angular dependence of the resonance field $\mathrm{B}_{\mathrm{r}}$.

\section{RESULTS}

Fig. 1 shows the hysteresis loops measured along the easy [100] and in-plane hard axis [110] in one of the continuous films as well as a loop measured with the field applied at $30^{\circ}$ from the easy axis. A very square loop is obtained for the easy axis, with a coercivity value of approximately $47 \mathrm{Oe}$ and remanence indistinguishable from the saturation magnetization $\mathrm{M}_{\mathrm{S}}$. The magnetization switches from $+\mathrm{M}_{\mathrm{S}}$ to $-\mathrm{M}_{\mathrm{S}}$ in a single high-susceptibility reversal process. In contrast, the hard-axis loop presents a single jump at about $32 \mathrm{Oe}$, followed by a low-susceptibility, reversible magnetization increase up to saturation that is reached at a field near 550 Oe. When measured along any direction intermediate between the easy and hard axes, the hysteresis loops present either one or two magnetization jumps. The inset in Fig. 1 presents the evolution with the angle $\theta$ between the applied field and the easy axis [100] of the switching field associated with these high susceptibility processes: a single switching field $\mathrm{H}_{\mathrm{S} 1}$ exists for loops measured at angles smaller than $10^{\circ}$ from an easy axis, whereas two switching fields, $\mathrm{H}_{\mathrm{S} 1}$ and $\mathrm{H}_{\mathrm{S} 2}$, appear for loops measured at $10^{\circ}<\theta<45^{\circ}$, as is the case of the loop measured at $30^{\circ}$ from the easy axis in the figure. The angular dependences of the switching fields and of the remanence corresponding to fields rotating $360^{\circ}$ in the sample plane were measured in order to check the biaxial anisotropy of the films. As can be seen in the azimuthal plots of Fig. 2, both the remanence and $\mathrm{H}_{\mathrm{S} 1}$ exhibit a pronounced

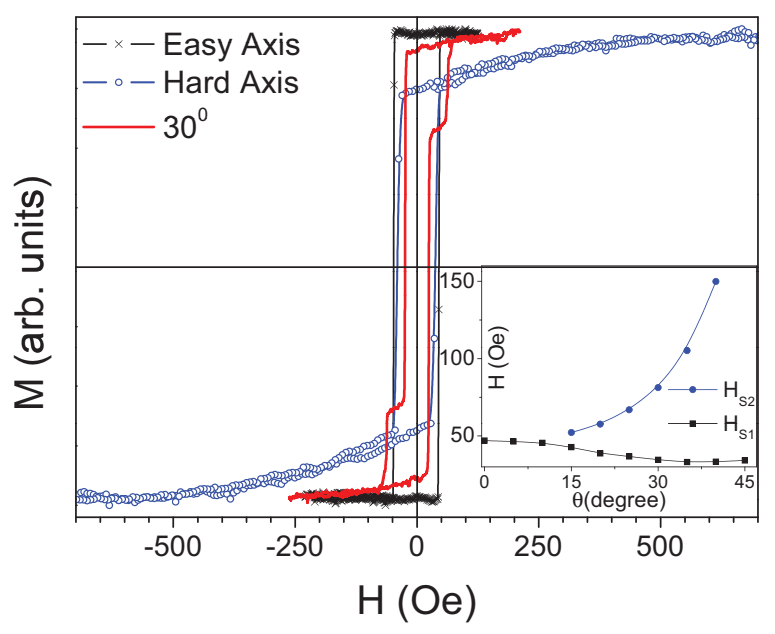

FIG. 1. Hysteresis loops of a continuous $\mathrm{Au} / \mathrm{Fe} / \mathrm{MgO}$ film measured along an easy [100] and hard in-plane [110] axis, respectively, and also with the field applied at $30^{\circ}$ from the easy axis. Inset: angular evolution of the switching fields. 

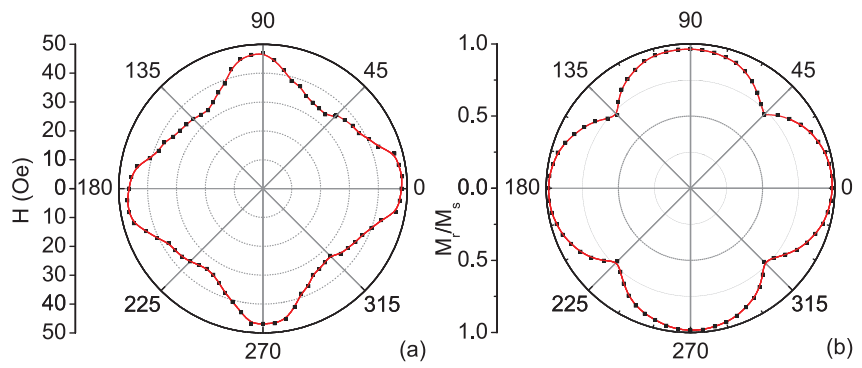

FIG. 2. Angular evolution of the switching field $\mathrm{H}_{\mathrm{S} 1}$ (a) and of the remanence-to-saturation ratio (b) of a continuous $\mathrm{Au} / \mathrm{Fe} / \mathrm{MgO}$ film.

four-fold symmetry with slight differences of the coercivity values measured along orthogonal directions. The basic characterization of the rf response was carried out by studying the in-plane angular dependence of the FMR spectra. The spectra were taken by rotating the sample around an axis perpendicular to the sample plane in $5^{\circ}$ steps, as depicted in Fig. 3(a) which shows that resonance peaks appear with a $90^{\circ}$ periodicity, just around the hard magnetocrystalline axes. The line sextet observed between 310 and $380 \mathrm{mT}$ is angle independent and was proven to be due to the paramagnetic signal of the substrate, obviously associated with $\mathrm{Mn}^{2+}$ ions in $\mathrm{MgO} .{ }^{19}$ The angular dispersion of the resonance around the hard axis was then resolved using $1^{\circ}$ steps, as can be seen in Fig. 3(b). The individual spectra consisted of a single resonance peak, as shown in the inset to this figure, which corresponds to the spectrum taken with the static field parallel to the [110] axis. The angular evolution of the resonance field $\mathrm{B}_{\mathrm{r}}$ around a full $360^{\circ}$ turn was studied by fitting the spectra using Dyson curves $^{20}$ evidencing the clear four-fold symmetry of the films, as can be seen in Fig. 3(c). The detailed analysis of the resonances shows that the field matching the resonance condition around the hard axis direction is $106 \mathrm{mT}$.

After the characterization of the films, six arrays of $0.5 \times 0.5 \mathrm{~mm}^{2}$ were fabricated on the $\mathrm{Au} / \mathrm{Fe} / \mathrm{MgO}$ films containing $100 \mu \mathrm{m}$ long stripes, with the widths $w$ and separations $d$ as indicated in Table I. The study of the angular dependence of the hysteresis processes revealed two types of magnetization mechanisms depending on the (in-plane) angle $\alpha$ between the long axis and the applied field. For angles up to about $60^{\circ}$, the magnetization reversal is based on a steep irreversible magnetization increase followed by a slow approach to saturation, with the coercivity following a $1 / \cos \alpha$ law. ${ }^{4} \mathrm{~A}$ different scenario occurs for large angles between the applied field and the axis of the stripes. Fig. 4 shows the hysteresis loops corresponding to an array of $200 \mathrm{~nm}$ wide stripes, measured with the applied field perpendicular to the long axis and also with the field $7^{\circ}$ off the perpendicular. When the applied field is perpendicular, the magnetization increases reversibly, until a jump up to saturation occurs at approximately $1.8 \mathrm{kOe}$, with some hysteresis around it. These features were common to all arrays, with the field required for saturation increasing from about $0.5 \mathrm{kOe}$ for the stripes $1000 \mathrm{~nm}$ wide to almost $2.5 \mathrm{kOe}$, for those $140 \mathrm{~nm}$ wide. A more complicated loop appears when the field is slightly off the perpendicular, typically for $70^{\circ}<\alpha<90^{\circ}$. In this case, the magnetization also increases slowly and suddenly drops giving rise to a hump, at approximately $1.4 \mathrm{kOe}$, for the array with $w=200 \mathrm{~nm}$, followed by a smooth approach to saturation (Fig. 4).

The study of the in-plane angular evolution of the FMR evidenced three types of resonances appearing along different directions and at different field ranges. As an example, Fig. 5 shows the spectra corresponding to an array with $w=d=200 \mathrm{~nm}$. A resonance with four-fold symmetry occurs at fields of $\leq 108 \mathrm{mT}$, the peaks appearing along the in-plane hard magnetocrystalline axes. The most intense and complex resonance appears when the field is applied along the $[\overline{1} 00]$ and [100] directions, i.e., perpendicular to the axis of the stripes, between approximately 150 and $200 \mathrm{mT}$. Finally, a very weak resonance can be observed at higher
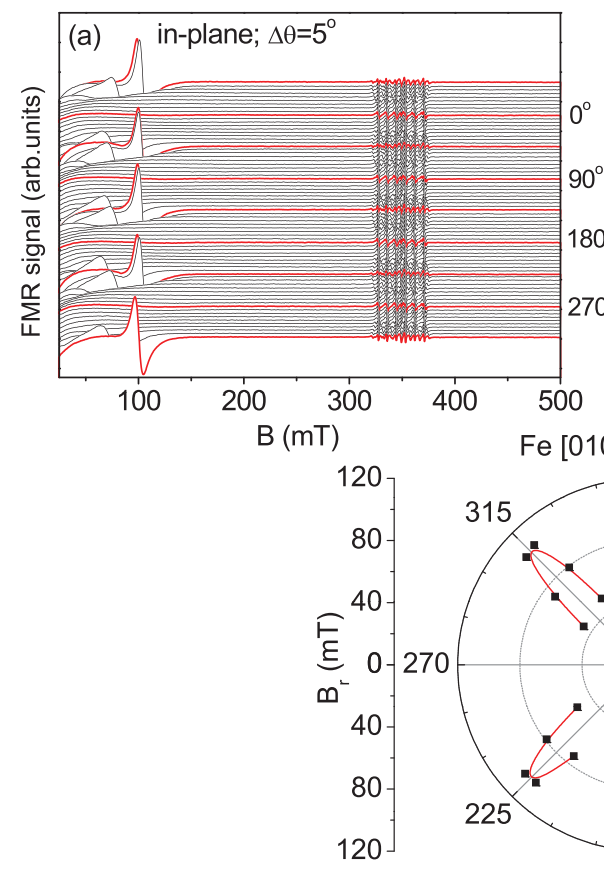

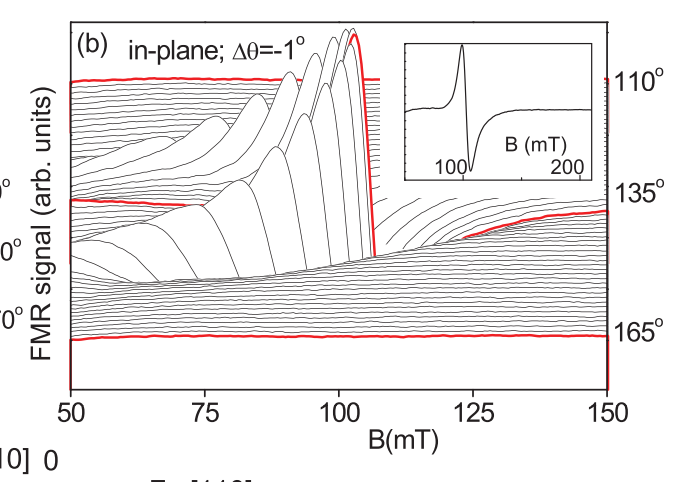<smiles>C1C2CC3CC1CC3C2</smiles> 
TABLE I. Width $w$ of the stripes and separation $d$ between them in the arrays.

\begin{tabular}{llllll}
\hline \hline Width $w(\mathrm{~nm})$ & 140 & 200 & 300 & 500 & 1000 \\
\hline$d=200 \mathrm{~nm}$ & & $\mathrm{x}$ & & $\mathrm{x}$ & \\
$d=500 \mathrm{~nm}$ & $\mathrm{x}$ & $\mathrm{x}$ & $\mathrm{x}$ & & $\mathrm{x}$ \\
\hline \hline
\end{tabular}

fields, around $400 \mathrm{mT}$, also for the spectra measured along the $[\overline{1} 00]$ and $[100]$ directions and partially overlapped with the substrate signal. All arrays exhibit a similar structure for the angular evolution of the spectra.

The low field resonances which appear along the [110] and [110] directions and at the same field value for all arrays are clearly coincident with those present in the continuous films. The hysteresis loops measured along these directions ${ }^{4}$ showed that, even in the case of the narrowest stripes, a field of $108 \mathrm{mT}$ is enough to drive the magnetization to saturation or very close to it and, as a consequence, we can consider that the low-field resonances correspond to a saturated mode, with the magnetization precessing around the applied field.

The intermediate field resonances appear at fields and angular ranges centred around $90^{\circ}$ and $270^{\circ}$, which correspond to the direction perpendicular to the axis of the wires. Figs. 6(a)-6(f) show a fragment of these resonances for all arrays. The resonances are angularly spread over a region that increases with the width of the stripes (Fig. 7) and are characterized by a complex structure, each spectrum having several peaks. The colour plots of Fig. 6 allow to observe the angular width of the resonances while the dots in the plots indicate the value of the resonance fields measured at each field orientation. The widest stripes present the widest resonance region as well as the most complex structure: the spectra of the array with $w=1000 \mathrm{~nm}$ cover a region that spreads over $90^{\circ}$, thus overlapping with the peaks centred around the hard magnetocrystalline axes and include up to six resonance peaks (some of them very weak) for some angles. On the contrary, the intermediate field resonances of the stripes $140 \mathrm{~nm}$ and $200 \mathrm{~nm}$ wide roughly extend over a $10^{\circ}$ range,

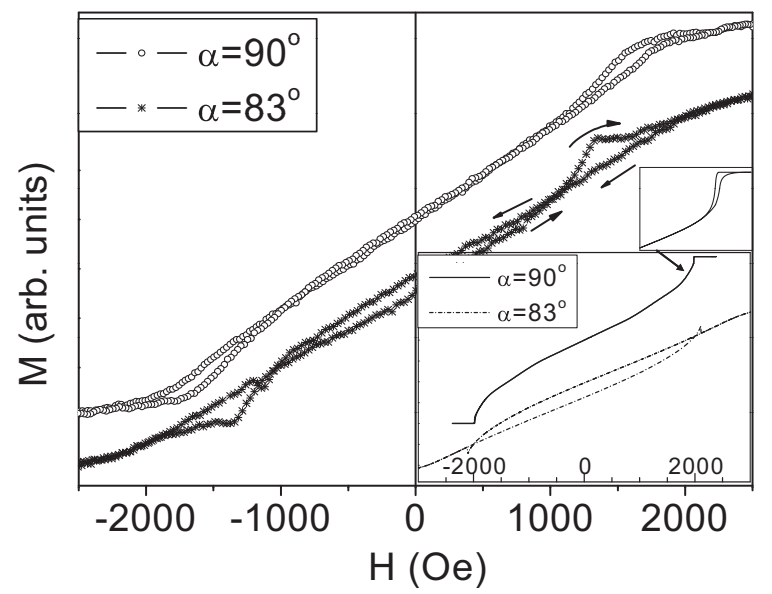

FIG. 4. Hysteresis loop of an array of $200 \mathrm{~nm}$ wide stripes, with the field applied in the film plane along the perpendicular to the long axis $\left(\alpha=90^{\circ}\right)$ and also $7^{\circ}$ off the perpendicular $\left(\alpha=83^{\circ}\right)$. Inset: loops calculated using the macrospin approximation.

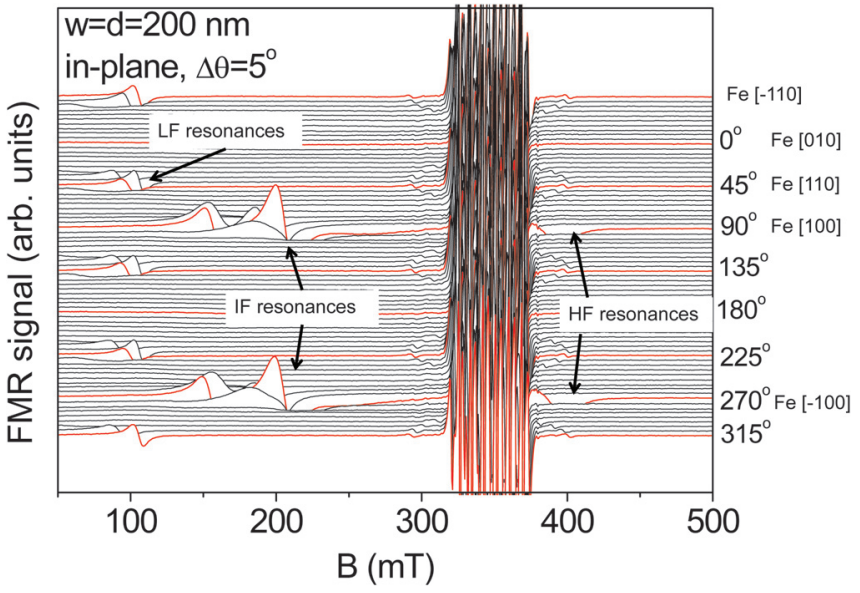

FIG. 5. In-plane angular evolution of the FMR spectra of an array with $w=d=200 \mathrm{~nm}$ showing three resonance regions at low (LF), intermediate (IF), and high (HF) fields. In this figure, the angles $0^{\circ}$ and $180^{\circ}$ correspond to the long axis of the stripes, whereas $90^{\circ}$ and $270^{\circ}$ correspond to the (inplane) perpendicular direction.

with just two resonance peaks for most angles. In the case of the two arrays with identical stripe width $w$ but different interstripe separations $d$, the structure of the intermediate resonance region is the same, with just an asymmetry around the perpendicular direction, probably due to a slight misalignment of the axis of the stripes with respect to the crystalline [100] axis. The individual spectra measured along the short axis of the stripes for all arrays can be seen in Fig. 8, in which the vertical dotted lines indicate the field required to saturate each array. All spectra have two or more peaks, although just one of them in each scan is above the onset of saturation. In the case of the two arrays with $w=200 \mathrm{~nm}$ but with different separations between the stripes, the spectra are essentially the same, with the two main peaks appearing at the same field value and with the same relative intensity and just a slight difference in the weakest one, which is hardly visible for the array with $d=200 \mathrm{~nm}$. By plotting the highest resonance field of each spectra, $\mathrm{B}_{\mathrm{r}}$, it can be seen that it increases with decreasing width (Fig. 9), following a $1 / w^{2}$ law (see inset) irrespectively of the separation between the stripes.

The features of the high-field resonances, which were observed with the applied field around the perpendicular to the long axis of the stripes, are strikingly different from those at low and intermediate fields. Figs. 10(a)-10(e) show the angular dependence of the spectra and the angular width of the resonance fields, whereas Fig. 11 exhibits the spectra along the perpendicular of the four arrays with stripes of different widths but identical separation between them, $d=500 \mathrm{~nm}$. Table II summarizes the correlation between the resonance parameters of these arrays (resonance field, peak amplitude and width, as well as angular width of the resonance region) and their morphology. The Fe coverage, which represents the percentage of the area of the array covered with $\mathrm{Fe}$ and scales as $w / w+d$, is proportional to the total amount of $\mathrm{Fe}$ in the array since all arrays have the same lithographed area. The density of the lateral surfaces, calculated as $2 / w+d$, represents the number of sides of the stripes per unit length measured along the (in-plane) direction 
(a)
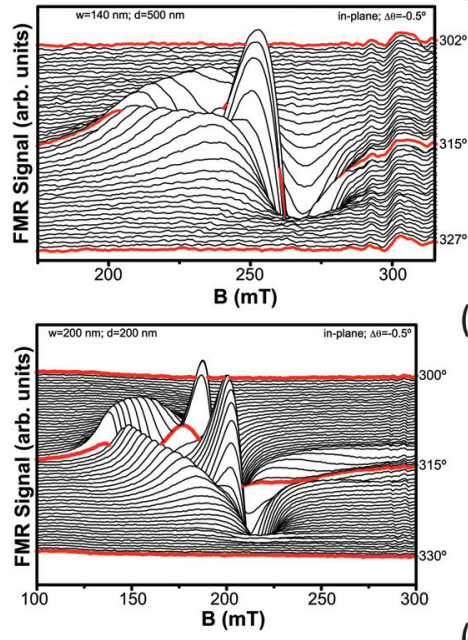

(c)

(b)
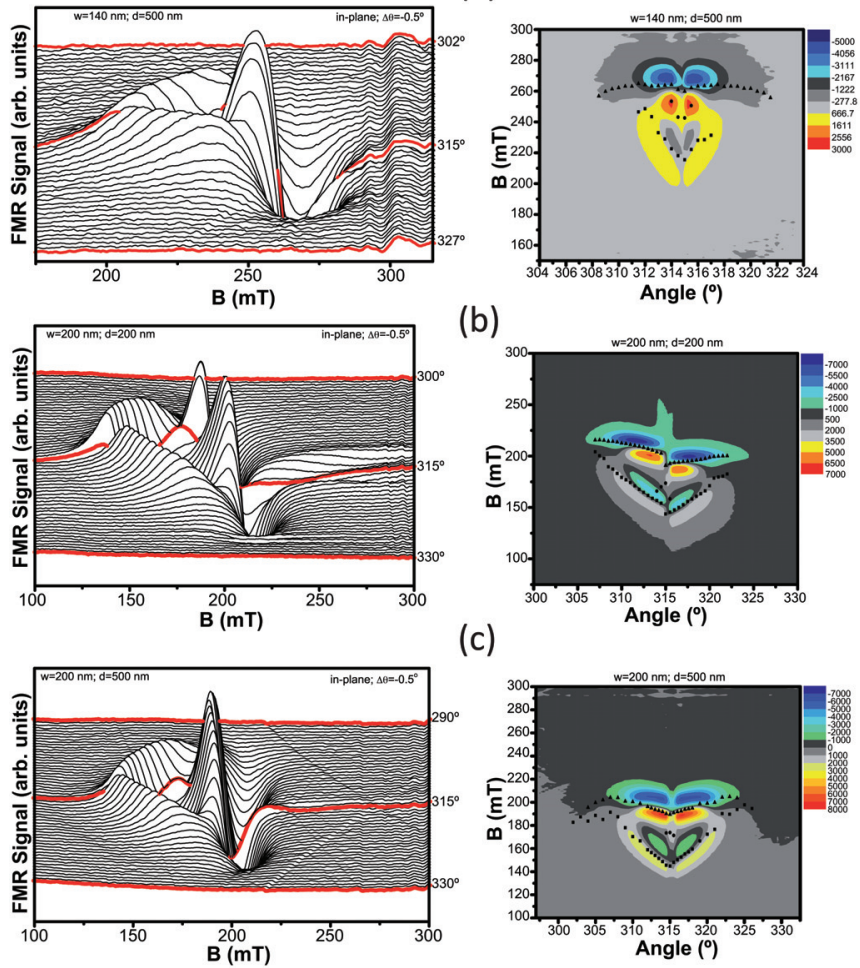

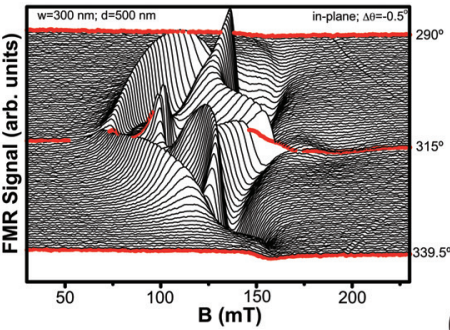

(d)

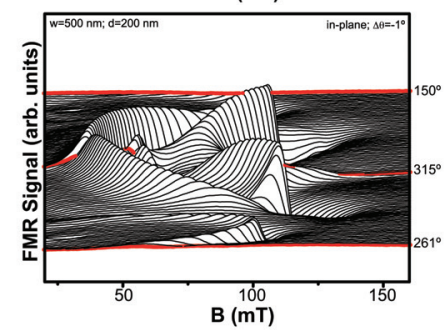

(e)
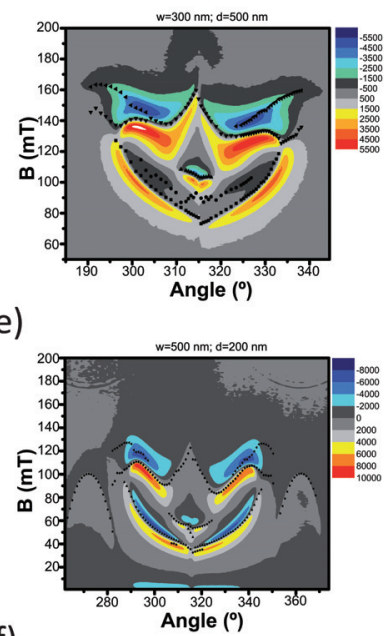

(f)
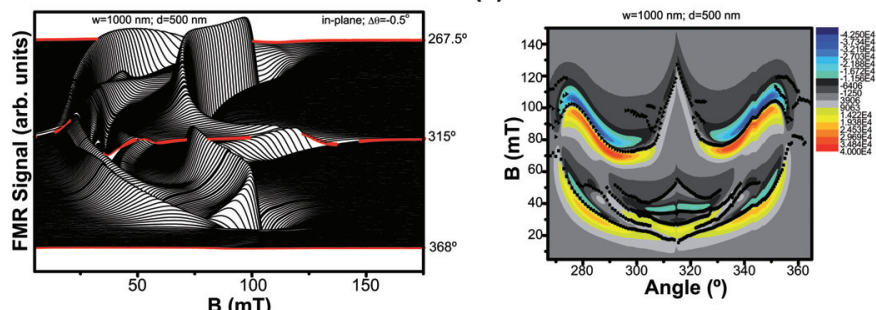

FIG. 6. Fragment of the intermediate field resonance region of all arrays: $w=140 \mathrm{~nm}(\mathrm{a}), w=d=200 \mathrm{~nm}(\mathrm{~b}), w=200 \mathrm{~nm}$ with $d=500 \mathrm{~nm}(\mathrm{c}), w=300 \mathrm{~nm}$ (d), $w=500 \mathrm{~nm}(\mathrm{e})$, and $w=1000 \mathrm{~nm}$ (f). The colour plots show the FMR signal over the background (grey), tendency to blue (red) indicates negative (positive) values over the background while the dots indicate the value of the resonance $\mathrm{B}_{\mathrm{r}}$. In these plots, the angle of $315^{\circ}$ corresponds to the wires' short axis direction.

perpendicular to the long axis. The angular width of the region, in which the high-field resonances can be observed, is almost the same for all arrays, viz., about $7^{\circ}$. The resonance field $\mathrm{B}_{\mathrm{r}}$ lies in a narrow band for all the arrays with $d=500 \mathrm{~nm}$, between 470 and $535 \mathrm{mT}$, with the highest values corresponding to the narrowest stripes. In contrast, the resonance of the stripes $200 \mathrm{~nm}$ wide and with $d=200 \mathrm{~nm}$ separation, which partially overlaps with the substrate EPR signal, takes place at a much lower field near $390 \mathrm{mT}$. Nohigh field resonance was observed for stripes $500 \mathrm{~nm}$ wide with $200 \mathrm{~nm}$ separation, which can be tentatively attributed to its overlap with the substrate signal. In what follows we shall present the data corresponding just to the arrays with $d=500 \mathrm{~nm}$. In a similar way to the intensity of the

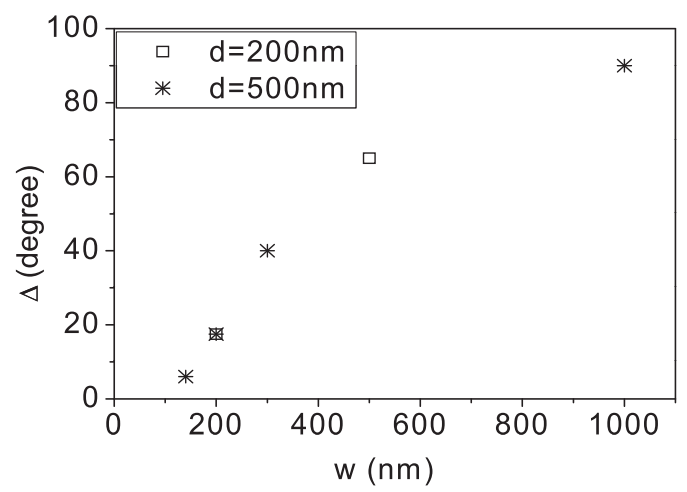

FIG. 7. Angular width $\Delta$ of the intermediate field resonances as a function of the stripe width. resonances, the peak width $\Delta \mathrm{B}$, measured as the peakto-peak distance, being around $50 \mathrm{mT}$, is very weakly dependent on the width of the stripes. The intensity of the resonances, measured as the peak-to-peak signal amplitude, is clearly higher for the narrowest stripes, 140 and $200 \mathrm{~nm}$ wide, being minimum for $w=300 \mathrm{~nm}$. The intensity obviously correlates with two morphological parameters. Taking into account that all arrays have the same total area, the Fe coverage (understood as the area percentage covered with

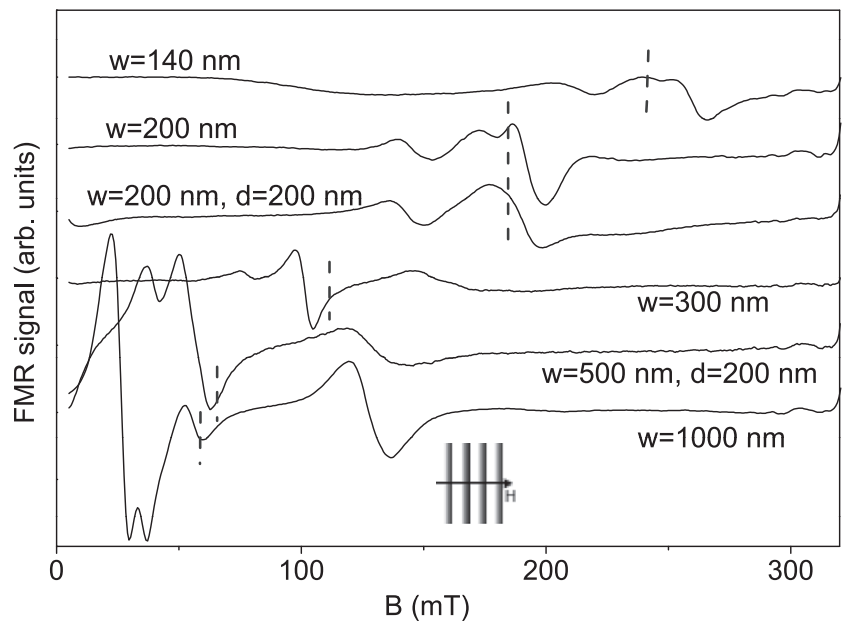

FIG. 8. Intermediate region of the FMR spectra of all arrays measured with the field applied in the sample plane perpendicular to the long axis of the stripes $(d=500 \mathrm{~nm}$ for all spectra unless otherwise specified). The dotted line indicates the field value required to saturate each array. 


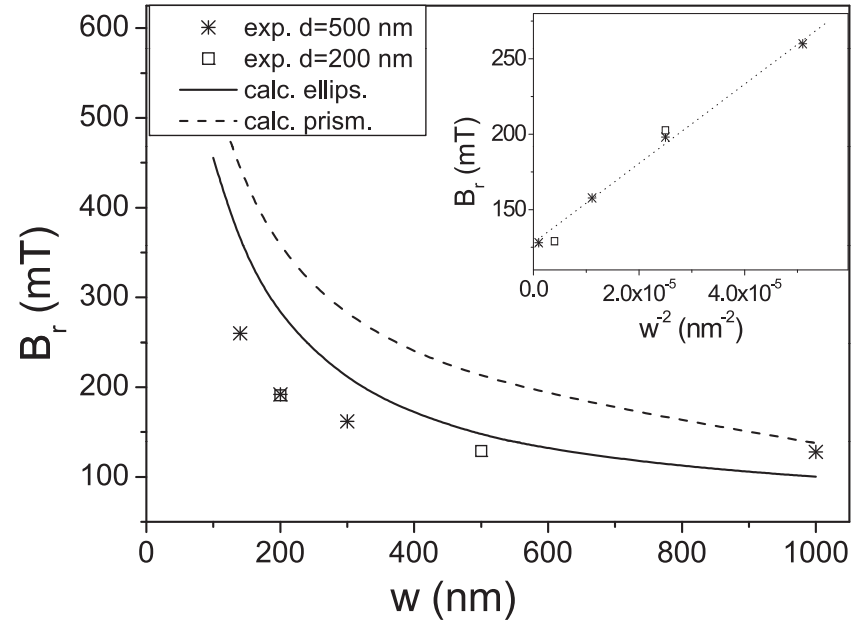

FIG. 9. Dependence of the highest resonance field, $B_{r}$, of the spectra shown in Fig. 8, on the stripe width. Inset: $1 / w^{2}$ dependence of $\mathrm{B}_{\mathrm{r}}$.

$\mathrm{Fe}$ ) and the density of lateral surfaces (representing the number of stripe sides per unit length measured along the perpendicular) are clearly higher for the arrays containing the narrow stripes. Thus, the intensity of the resonance does not increase with the total amount of Fe in the array but with the number of lateral surfaces.

\section{DISCUSSION}

As a first point, we will analyze the hysteresis processes and FMR measurements of the continuous Fe film on which the arrays were then lithographed. The hysteresis processes measured along the easy axis of the continuous films, based on a single, very narrow switching event, are typical of the nucleation and propagation sequence caused by the generation of one or just a few reversed coalescing nuclei that sweep the whole film, with no effective hindrances, when the field reaches the coercive force value. ${ }^{5,7}$ The remanence of the loops measured along the in-plane hard axis, $0.7 \mathrm{M}_{\mathrm{S}}$, confirms that in the absence of any applied field the magnetization lies along the easy axis (at $45^{\circ}$ with respect to the hard axis), while the field required to reach saturation, 550 $\mathrm{Oe}$, agrees well with anisotropy field of Fe. ${ }^{5}$ The existence or either one or two switching fields, depending on the orientation of the applied field relative to the crystalline axes, is also a signature of single-crystalline $\mathrm{Fe}(001)$ films, the second jump being associated, when it is present, with an intermediate energy minimum due to the biaxial nature of the anisotropy. This has been widely studied in many previous works: both calculations and experimental results reported in the literature about Fe (001) single-crystalline films, prepared by different techniques, have evidenced that the main switching field $\mathrm{H}_{\mathrm{S} 1}$ (corresponding to the largest magnetization jump) decreases while $\mathrm{H}_{\mathrm{S} 2}$ (corresponding to the smallest jump) increases when the applied field approaches the hard axis, in agreement with our results (see inset to Fig. 1). ${ }^{5,7}$ An important issue lies in the in-plane angular dependence of the coercivity and remanence (Fig. 2) and of the FMR spectra (Fig. 3), which evidence the clear four-fold symmetry of the films, with weak traces of other superim-
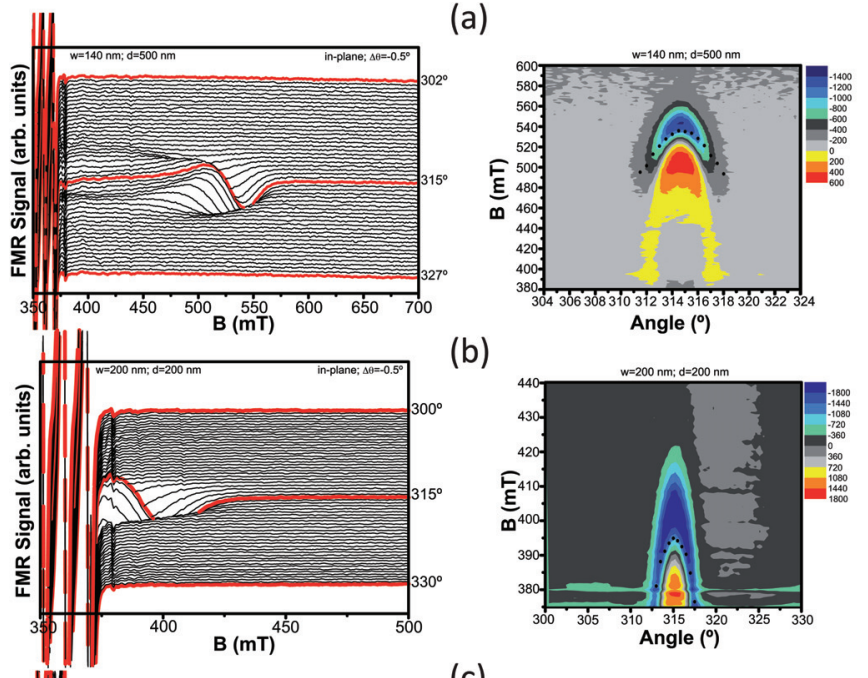

(c)
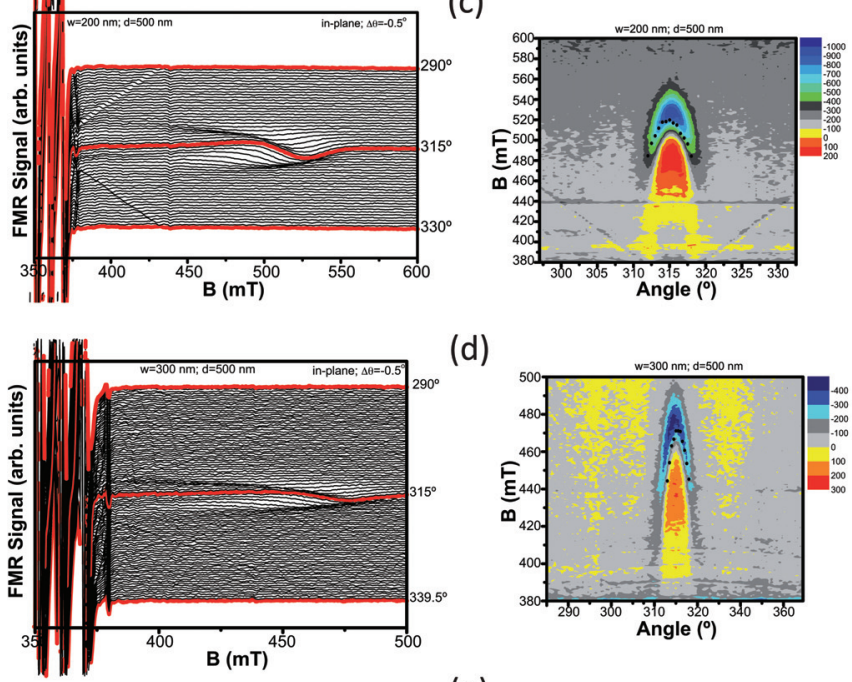

(d)

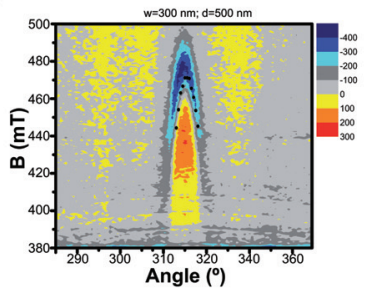

(e)
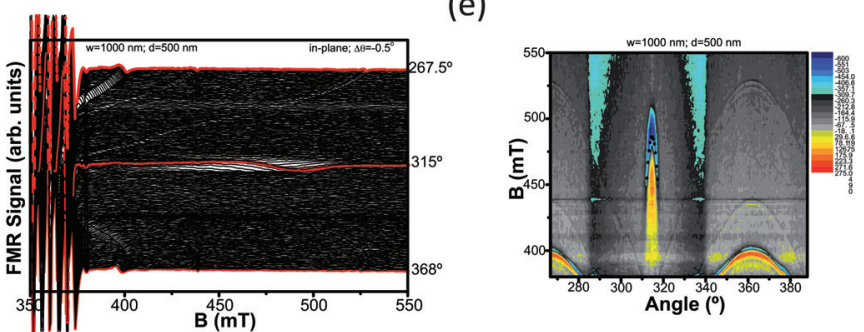

FIG. 10. Detail of the high-field-resonance region of all arrays: $w=140 \mathrm{~nm}$ (a), $w=d=200 \mathrm{~nm}$ (b), $w=200 \mathrm{~nm}$ with $d=500 \mathrm{~nm} \mathrm{(c),} w=300 \mathrm{~nm}$ (d), and $w=1000 \mathrm{~nm}$ (e). The colour plots show the FMR signal over the background (grey), tendency to blue (red) indicates negative (positive) values over the background while the dots indicate the value of the resonance field; the short axis direction corresponds to $315^{\circ}$ in these plots.

posed anisotropy contribution. This minor, difficult-toobserve difference in the coercivity values measured along orthogonal directions could be due to the difficulty to fix the spot in the exact centre of the rotation axis during the MOKE measurements. These differences do not alter the main conclusion that the films can be treated in the frame of purely biaxial anisotropy. The FMR spectra allowed a precise determination of the anisotropy constant of the films. Taking into account that the resonance peaks appear at $106 \mathrm{mT}$, well above the field required to saturate the films along the hard axis, we can assure that the resonance 


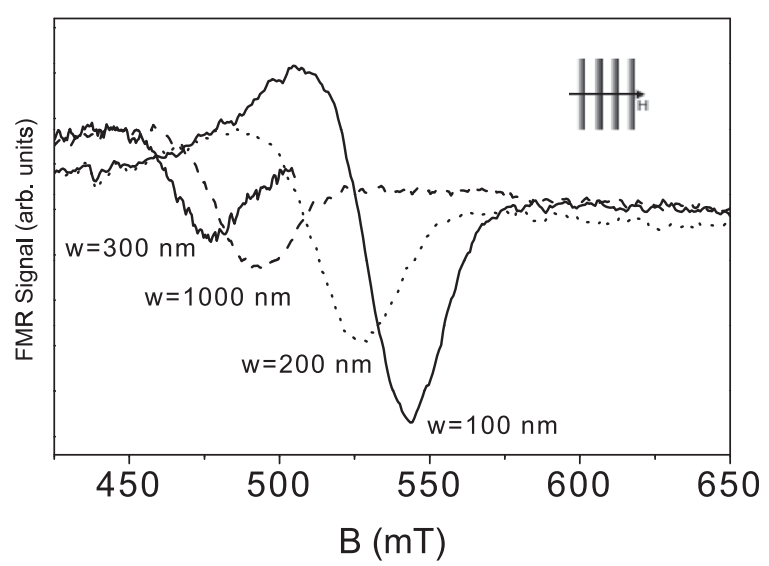

FIG. 11. High-field spectra measured with the field applied in the sample plane along the perpendicular to the stripes, corresponding to the four arrays with $d=500 \mathrm{~nm}$.

corresponds to the so-called aligned mode, i.e., the magnetization vector is uniform and precesses around the applied field direction. Under these conditions, the resonant field $\mathrm{B}_{\mathrm{r}}$ can be calculated by means of the general formalism of Smit and Beljers: ${ }^{21}$

$$
\left(\frac{\omega}{\gamma}\right)^{2}=\frac{1}{M_{S}^{2} \sin ^{2} \theta}\left(F_{\theta \theta} F_{\varphi \varphi}-F_{\theta \varphi}^{2}\right)
$$

in which $\theta$ and $\varphi$ represent the polar and azimuthal angles of the magnetization with respect to a given set of coordinate axes, $F_{\theta \varphi}, F_{\theta \theta}$, and $F_{\varphi \varphi}$ are the second derivatives of the free energy with respect to these angles, and $\omega, \gamma$, and $\mathbf{M}_{\mathbf{S}}$ are the resonance angular frequency, the gyromagnetic ratio and the saturation magnetization, respectively. In the specific case of single-crystalline $\mathrm{Fe}(001)$ thin films saturated along either the easy (100) or hard (110) axis, the resonance condition can be calculated from Eq. (1) as

$$
\left(\frac{\omega}{\gamma}\right)^{2}=\left(B_{r}-\frac{2 K}{M_{S}}\right)\left(B_{r}+\mu_{0} M_{S}+\frac{K}{M_{S}}\right),
$$

when the field is applied along the hard axis and as

$$
\left(\frac{\omega}{\gamma}\right)^{2}=\left(B_{r}+\frac{2 K}{M_{S}}\right)\left(B_{r}+\mu_{0} M_{S}+\frac{K}{M_{S}}\right),
$$

when it is applied along the easy axis. In these expressions, $\mathrm{B}_{\mathrm{r}}$ is the resonance field corresponding to $\omega, \mathrm{K}$ represents the anisotropy constant of $\mathrm{Fe}\left(\mathrm{K}=4.8 \cdot 10^{4} \mathrm{~J} \cdot \mathrm{m}^{-3}\right), \mathrm{M}_{\mathrm{S}}$ its

\begin{tabular}{|c|c|c|c|c|c|c|}
\hline $\begin{array}{l}\text { Width } \\
(\mathrm{nm})\end{array}$ & $\begin{array}{c}\mathrm{B}_{\mathrm{r}} \\
(\mathrm{mT})\end{array}$ & $\begin{array}{l}\text { Peak-to-peak } \\
\text { amplitude } \\
\text { (arb. units) }\end{array}$ & $\begin{array}{c}\text { Peak-to-peak } \\
\text { width (mT) }\end{array}$ & $\begin{array}{l}\text { Angular } \\
\text { width } \\
\text { (deg) }\end{array}$ & $\begin{array}{l}\text { Lateral } \\
\text { density } \\
\left(\mathrm{nm}^{-1}\right)\end{array}$ & $\begin{array}{c}\mathrm{Fe} \\
\text { coverage } \\
(\%)\end{array}$ \\
\hline 140 & 520 & 1910 & 52 & 7 & $3.12 \times 10^{-3}$ & 22 \\
\hline 200 & 500 & 1460 & 59 & 8 & $2.86 \times 10^{-3}$ & 28 \\
\hline 300 & 460 & 625 & 44 & 8 & $2.50 \times 10^{-3}$ & 37 \\
\hline 1000 & 470 & 795 & 57 & 7 & $1.33 \times 10^{-3}$ & 67 \\
\hline
\end{tabular}

TABLE II. Correlation between the high-field resonance parameters and the morphology of the arrays with the stripes separated by $d=500 \mathrm{~nm}$. saturation magnetization $\left(\mathrm{M}_{\mathrm{S}}=1.71 \cdot 10^{6} \mathrm{~A} \cdot \mathrm{m}^{-1}\right)$ and $\gamma$ is the gyromagnetic ratio $\left(\gamma=1.759 \cdot 10^{11} \mathrm{~T}^{-1} \mathrm{~s}^{-1}\right){ }^{22}$ Using these values in combination with the frequency of the microwave radiation in our experimental setup, $9.85 \mathrm{GHz}$, the calculated resonant field along the hard axis results to be $107.5 \mathrm{mT}$, in excellent agreement with the experimental value. When the applied field is parallel to the easy axis, the resonant field calculated from Eq. (3) is null and, as a consequence, the resonance condition cannot be achieved due to the remanent field of the electromagnet used to generate the static field. It is important to remark that no lower field resonances appear in our spectra when sweeping the static field from $1.5 \mathrm{~T}$ to $0 \mathrm{~T}$. The absence of low-field resonance peaks indicates that the anisotropy contributions different from the magnetocrystalline term are negligible in these measurements, in good agreement with the perfect four-fold symmetry of the coercivity and of the remanence. In contrast, many Fe films reported in the literature, either deposited on $\mathrm{MgO}$ or semiconductor substrates, present an extrinsic contribution to the anisotropy of structural origin (uniaxial in most cases), usually due to morphological features of the substrate, such as steps or miscuts, to the elastic stresses generated by the lattice mismatch at the interface or to the growth mode of the Fe. ${ }^{6,8-11}$ The relative intensity of this contribution tends to decrease with increasing thickness of the films because of their interfacial origin, although it does not become negligible even for films as thick as tens of nanometers. ${ }^{12,23}$ The extrinsic anisotropy usually induces significant modifications of the hysteresis properties, breaking the four-fold symmetry of the remanence and coercivity, and it might even give rise to demagnetization processes with two irreversible steps in the loops measured along the easy axes. ${ }^{12}$ Regarding its influence on the FMR spectra, it also breaks the four-fold symmetry, and induces resonance peaks that are not expected either along the hard axes or along different directions. We can conclude that the four-fold in-plane anisotropy of our $\mathrm{Au} / \mathrm{Fe} / \mathrm{MgO}$ films is just due to the magnetocrystalline anisotropy contribution, as evidenced by the analysis of the hysteresis behaviour and the FMR studies, which is a clear indication of the excellent single-crystalline nature of the continuous films on which the stripes were lithographed.

Regarding the stripes, their hysteresis properties are based on two different mechanisms. At low angles between the applied field and the (long) axis of the stripes $\left(\alpha<60^{\circ}\right)$ the loops are characterized by a single, irreversible magnetization jump followed by a reversible approach to the saturation region, as evidenced in a previous work, ${ }^{4}$ which also showed that the angular dependence of the coercivity follows a $1 / \cos \alpha$ law, typical of wall pinning. Both MFM images and indirect evidences have shown that the pinning takes place at the irregularities along the sides of the stripes and that it is basically independent of the lithography techniques employed to fabricate them. ${ }^{4,6,7}$ On the contrary, when the applied field is close to the perpendicular to the (long) axis of the stripes, the magnetization proceeds through very smooth magnetization rotations up to high fields with small irreversible jumps appearing when the magnetization vector is very close to the applied field. This is due to an irreversible jump of the magnetization from one minimum to another 
(the energy landscape is relatively complex, with several minima evolving in different ways as the field increases) and is associated with a change of sign in the transverse magnetization component (in-plane, perpendicular to the applied field). These high-angle magnetization processes can be described in terms of uniform magnetization configurations, i.e., of a macrospin, by taking into account just the Zeeman, magnetocrystalline and magnetostatic energy terms with a very good qualitative and quantitative agreement between the calculations and the experimental results: the inset of Fig. 4 shows the hysteresis loops calculated, with the field applied either perpendicularly or $7^{\circ}$ out of the perpendicular, for a stripe $200 \mathrm{~nm}$ wide by using the intrinsic parameters of $\mathrm{Fe}$, for $\mathrm{M}_{\mathrm{S}}, \mathrm{K}$ and the demagnetizing factors calculated from Ref. 24. As can be seen, both calculated loops reproduce the main features of the experimental loops, with the irreversible jump and the hump occurring at field values very close to those experimentally measured. Although not shown in the figure, the loops, in which the transverse component of the magnetization is plotted versus the applied field, also evidence a very good agreement between the experiments and the calculations.

An important issue related to the hysteresis of the arrays is that of the dipolar interactions and of the possible influence of the lithography route employed. Regarding the dipolar interactions, no significant differences were obtained when measuring the loops, either at low or high angles, for the arrays with similar width of the stripes but different separations $d$ between them. This is true not only for the arrays studied in this work but also for a different set of arrays fabricated using different lithography techniques (focused ion beam and electron beam lithography with positive resist) and with a broader spectrum of separations. ${ }^{4}$ The fact that, for stripes of the same width, the coercivity of all arrays was essentially the same and the characteristic features observed for the high-angle loops (irreversible jumps and humps) occurred at very close field values, irrespectively of their separation, are a clear indication that the dipolar interactions, at least for interstripe separations of the order of or above $200 \mathrm{~nm}$, are negligible, and that the defects generated during the lithography processes have little influence on the hysteresis parameters of the arrays.

The study of the angular evolution of the FMR spectra of the arrays has evidenced three different types of resonances. Those occurring at low fields appear when the field is applied along the in-plane hard magnetocrystalline directions, [110] and [110], and yield a $\mathrm{B}_{\mathrm{r}}$ value close to $108 \mathrm{mT}$ for all arrays, i.e., coincident with that measured in the continuous films, irrespectively of the width of the stripes. This suggests that these modes are linked to the magnetocrystalline anisotropy which is not essentially modified during the lithography process. The intermediate field resonances present a very complex angular dependence and a very rich structure. These resonances appear when the (in-plane) field is near the perpendicular to the long axis of the stripes-the hard axis from the magnetostatic point of view-and all spectra exhibit several peaks, although just the one occurring at the highest field value corresponds to the saturated mode, as evidenced in Fig. 8. The calculations of the magnetization processes of the arrays have demonstrated that the magnetization is essentially homogeneous prior to saturation and that it is stabilized in several energy minima before the final jump to saturation. It is quite likely that the peaks appearing in the spectra at fields just below saturation are due to the oscillations of the magnetization around these minima. Several issues are important when considering the peaks associated with the saturated mode; the first one is that all resonance field values fall within the same $1 / w^{2}$ plot, irrespectively of the separation between the stripes (Fig. 9). In addition to it, the two arrays with stripes $200 \mathrm{~nm}$ wide, but with separation of either $200 \mathrm{~nm}$ or $500 \mathrm{~nm}$ between them, have the same resonant field. This confirms the negligible influence of the dipolar interactions already inferred from the study of the hysteresis processes. The value of the resonance field can be calculated from Eq. (1) by taking into account the Zeeman, magnetocrystalline, and magnetostatic energy terms in the free energy. By considering the demagnetizing factors along the long $(x)$, short $(y)$, and perpendicular to the plane (z) axes- $N_{x}, N_{y}$, and $N_{z}$, respectively-the magnetostatic energy term can be written as

$$
F_{\text {mag }}=\frac{1}{2} \mu_{0}\left(N_{x} M_{x}^{2}+N_{y} M_{y}^{2}+N_{z} M_{z}^{2}\right),
$$

and Eq. (1) becomes

$$
\begin{aligned}
\left(\frac{\omega}{\gamma}\right)^{2}= & {\left[\left(B_{r}\right)^{2}+\left(B_{r}\right)\left[\frac{4 K}{M_{S}}+\mu_{0} M_{S}\left(N_{x}+N_{z}-2 N_{y}\right)\right]\right.} \\
& \left.+\left(\mu_{0} M_{S}\right)^{2}\left(N_{z}-N_{y}\right)\left(N_{x}-N_{y}\right)+\left(\frac{2 K}{M_{S}}\right)^{2}\right] .
\end{aligned}
$$

Two different sets of demagnetizing factors can be introduced in Eq. (5), corresponding either to long rods with ellipsoidal cross section ${ }^{24}$ or to long prisms with rectangular cross section. ${ }^{25}$ Fig. 10 presents the width dependence of the experimental $B_{r}$ values compared to the calculated curves using the previously mentioned $\mathrm{Fe}$ parameters and the demagnetizing factors for stripes $24 \mathrm{~nm}$ thick. Although the experimental and calculated curves exhibit the same qualitative behaviour, the experimental data fall well below the curve calculated using the prism demagnetizing factors and just slightly below that calculated using the ellipsoidal rod ones. Micromagnetic simulations and experimental evidences have shown that, in the case of transversally magnetized micron-sized permalloy stripes, the equilibrium magnetization configurations include zones near the edges of the stripes with a large component of the magnetization parallel to the axis of the stripes. ${ }^{16,17,26-29}$ The eventual presence of these not fully saturated zones near the edges of the stripes could possibly reduce the transverse dipolar fields involved in the resonance conditions, thus giving rise to a decrease of the resonance field of the stripes. It is important to note that, although the resonances occur after the wires have reached saturation, as indicated by the dotted lines in Fig. 8, the resonance of the widest wires takes place at fields well above the onset of saturation, whereas those of the narrowest ones occur just after or even at the onset of saturation. Due to this, the non-saturated regions at the edges could be larger for the 
latter than for the former, which could explain why the difference between calculated and experimental resonance field values increases with decreasing width of the stripes.

The high-field resonances, in contrast, present a very different behaviour: all resonances consist of a single peak, visible just when the (in-plane) field is oriented along the perpendicular to the long axis of the stripes and in a region of approximately $7^{\circ}$ around this direction, irrespectively of the width of the stripes. In the case of the $200 \mathrm{~nm}$ wide stripes, the resonance field is strongly dependent on the interstripe separation, the resonance field of the closer stripes $(d=200 \mathrm{~nm})$ being well below that of the arrays with the well separated stripes $(d=500 \mathrm{~nm})$. The fact that the resonance of the array with $w=500 \mathrm{~nm}$ and $d=200 \mathrm{~nm}$ is probably overshadowed by the substrate signal is also consistent with the decrease of the resonance field when the stripes are relatively close to one another. However, for the arrays with well separated stripes, the relative variation of the resonance field with the stripe width is roughly $13 \%$, while the peak-topeak linewidth and the angular width of the resonance region remain essentially constant, independently of the width of the stripes. The correlation between the morphological parameters of the arrays and the intensity of the resonances (Table II) clearly show that the amplitude of the peaks is not linked to the whole volume of the stripes-because the resonances of the arrays with the lowest amount of $\mathrm{Fe}$ have the most intense peaks - but to the density of lateral edges. Thus, the experimental evidences point out that the high-field resonances appear due to oscillations localized near the edges of the stripes which are very sensitive to the dipolar fields. The presence of strong non-uniform dipolar fields near the edges of transversally magnetized stripes usually leads to the appearance of edge-localized precession modes, which-in the case of permalloy stripes-have been extensively studied either theoretically or experimentally, by means of Brillouin light scattering (BLS), FMR and time-resolved Kerr magnetometry. ${ }^{16-18,26-29}$ These precession modes or localized spin waves are quite sensitive to the internal fields and to the specific conditions of the edges of the stripes. As a general trend, the resonance graphs of the stripes (resonance frequency as a function of the applied field) have at least two branches corresponding, respectively, to the bulk (uniform resonance) and edge (localized spin waves) modes. The first one lies above the second, which implies that, for a fixed resonance frequency, larger fields are required to excite the edge modes. ${ }^{16,17}$ In the case of the high-field resonances observed in our epitaxial Fe stripes, an important issue is the reduced resonance field of the array with $w=200 \mathrm{~nm}$ and $d=200 \mathrm{~nm}$ with respect to the array with identical stripe width but with $d=500 \mathrm{~nm}$. The dipolar interactions (when the samples are saturated in the transverse direction) give rise to lower dipolar fields in the edges of the closest stripes, which are probably the reason of the decrease in the resonance field. On the contrary, the weak dependence of the resonance field on the width of the stripes in the arrays with $d=500 \mathrm{~nm}$ indicates that the resonance conditions are essentially dependent on the local fields at the edges. Thus, all experimental facts suggest that the high-field resonances of our epitaxial Fe stripes could be ascribed to edge-localized spin wave oscillations.

\section{v. CONCLUSIONS}

We have carried out a combined MOKE and FMR study of the magnetic properties of a series of epitaxial $\mathrm{Fe}(001) /$ $\mathrm{MgO}(001)$ films and arrays of stripes with submicron widths and separations. The FMR studies and the analysis of the angular evolution of the hysteretic parameters have evidenced that the four-fold symmetry of the films is essentially due to the magnetocrystalline anisotropy, with negligible contribution of other anisotropy sources possibly arising from structural factors related either to the growth mode or to magnetoelastic effects. The use of a large series of arrays of stripes with different widths and separations has allowed to study the competition between the magnetocrystalline and shape anisotropies in the magnetization processes as well as the resonance regime linked to each type of anisotropy, as well as the role of the interactions in both the magnetization processes and the FMR. The magnetization processes can be described in terms of the magnetization reversal of a macrospin in a good agreement between experiments and calculations, with non-significant effects due to the dipolar interactions between the stripes. The FMR presents three resonance regimes in different field ranges. The high- and intermediate-field resonances correspond to uniform bulk modes that can be assigned to oscillations around the hard magnetocrystalline and hard in-plane shape axes, respectively. These resonances are not sensitive to the dipolar interactions between the stripes, which results in a good agreement when comparing the measured and calculated resonance field values. The high-field resonances are very weak and are associated with localized edge modes, probably due to the highly inhomogeneous dipolar fields at the lateral sides of the stripes. As a consequence, these resonances are extremely sensitive to the dipolar interactions between the stripes. As a final conclusion, the general agreement between the experimental and calculated hysteretic and FMR parameters confirms the high crystalline quality of the films and stripes, which allows to customize their magnetic behaviour for implementation in actual devices.

\section{ACKNOWLEDGMENTS}

This work has been carried out under the financial support of the Spanish MICINN (Grant Nos. MAT200766719-C03, MAT2010-18432, MAT2010-21088-C03-03 and Acción Integrada HP2007-0115), of the FCT project PEst-c/CTM/LA0025/2011 and of the Acção Integrada Luso-Espanhola no. E-95/08.

\footnotetext{
${ }^{1}$ V. Korenivski, J. Magn. Magn. Mater. 215-216, 800 (2000).

${ }^{2}$ N. Cramer, D. Lucic, R. E. Camley, and Z. Celinski, J. Appl. Phys. 87, 6911 (2000).

${ }^{3}$ S. S. P. Parkin, M. Hayashi, and L. Thomas, Science 320, 190 (2008).

${ }^{4}$ E. Paz, F. Cebollada, F. J. Palomares, F. García-Sánchez, and J. M. González, Nanotechnology 21, 255301 (2010).

${ }^{5}$ F. Cebollada, A. Hernando-Mañeru, A. Hernando, C. Martínez-Boubeta, A. Cebollada, and J. M. González, Phys. Rev. B 66, 174410 (2002); and references therein.

${ }^{6}$ C. Hassel, F. M. Römer, R. Meckenstock, G. Dumpich, and J. Lindner, Phys. Rev. B 77, 224439 (2008).

${ }^{7}$ U. Ebels, A. O. Adeyeye, M. Gester, R. P. Cowburn, C. Daboo, and J. A. C. Bland, Phys. Rev. B 56, 5443 (1997).
} 
${ }^{8}$ D. Spoddig, C. Urban, D. You, U. Köhler, and R. Meckenstock, J. Appl. Phys. 99, 08 J704 (2006).

${ }^{9}$ D. Spoddig, U. Köhler, M. Haak, M. Kneppe, T. Schmitte, A. Westphalen, K. Theis-Bröhl, R. Meckenstock, D. You, and J. Pelzl, Superlattices Microstruct. 43, 180 (2008).

${ }^{10}$ K. Postava, H. Jaffres, A. Schuhl, F. Nguyen Van Dau, M. Goiran, and A. R. Fert, J. Magn. Magn. Mater. 172, 199 (1997).

${ }^{11}$ Kh. Zakeri, Th. Kebe, J. Lindner, and M. Farle, J. Magn. Magn. Mater. 299, L1 (2006).

${ }^{12}$ R. Meckenstock, D. Spoddig, K. Himmelbauer, H. Krenn, M. Doi, W. Keune, Z. Frait, and J. Pelzl, J. Magn. Magn. Mater. 240, 410 (2002).

${ }^{13}$ K. H. Zakeri, T. H. Kebe, J. Lindner, C. Antoniak, M. Farle, K. Lenz, T. Tolinski, and K. Baberschke, Phase Trans. 79, 793 (2006).

${ }^{14}$ J. Jorzick, S. O. Demokritov, C. Mathieu, B. Hillebrands, B. Bartenlian, C. Chappert, F. Rousseaux, and A. N. Slavin, Phys. Rev. B 60, 15194 (1999).

${ }^{15}$ H. Puszkarski, M. Krawczyk, and J.-C. S. Lévy, J. Appl. Phys. 101, 024326 (2007).
${ }^{16}$ B. B. Maranville, R. D. Michaels, S. A. Kim, W. L. Johnson, C. A. Ross, and J. Y. Cheng, J. Appl. Phys. 99, 08 C703 (2006).

${ }^{17}$ M. Zhu and R. D. McMichael, J. Appl. Phys. 109, 043904 (2011).

${ }^{18}$ M. Zhu and R. D. McMichael, J. Appl. Phys. 107, 103908 (2010).

${ }^{19}$ W. Low, Phys. Rev. 105, 793 (1957).

${ }^{20}$ F. J. Dyson, Phys. Rev. 98, 349 (1955).

${ }^{21}$ J. Smit and H. G. Beljers, Phillips Res. Rep. 10, 113 (1955).

${ }^{22}$ S. Chikazumi, Physics of Magnetism (Krieger, Malabar FL, 1978), p. 47.

${ }^{23}$ J. L. Costa-Kramer, J. L. Menéndez, A. Cebollada, F. Briones, D. García, and A. Hernando, J. Magn. Magn. Mater. 210, 341 (2000).

${ }^{24}$ A. Aharoni, J. Appl. Phys. 83, 3432 (1998).

${ }^{25}$ J. A. Osborn, Phys. Rev. 67, 351 (1945).

${ }^{26}$ C. Bayer, S. O. Demokritov, B. Hillebrands, and A. N. Slavin, Appl. Phys. Lett. 82, 607 (2003).

${ }^{27}$ C. Bayer, J. P. Park, H. Wang, C. E. Campbell, and P. A. Crowell, Phys. Rev. B 69, 134401 (2004)

${ }^{28}$ M. Bailleul, D. Olligs, and C. Fermon, Phys. Rev. Lett. 91, 137204 (2003).

${ }^{29}$ B. B. Maranville, R. D. Michaels, and d. W. Abraham, Appl. Phys. Lett. 90, 232504 (2007) 
Journal of Applied Physics is copyrighted by the American Institute of Physics (AIP). Redistribution of journal material is subject to the AIP online journal license and/or AIP copyright. For more information, see http://ojps.aip.org/japo/japcr/jsp 